\title{
Pressure Sensor Behavioral Search: Simulation Research for Aerospace Application
}

\author{
Vaishnavi Wagh, Shefali Sonavane, Chandan Kapoor
}

\begin{abstract}
Pressure sensors are used to monitor the performance characteristics of various systems within the appliance industry. Temperature, humidity altitude are the significant parameters to get the right information about atmospheric pressure. These geographical constraints affect the value of pressure. The pressure sensors are designed in such way that it should give correct information about all atmospheric changes. Micro Electro Mechanical System (MEMS) having piezoresistive pressure sensor has a various chip structure and sensing element. This chip structure helps to get various sensitivity results. Right now there are no results regarding impact of various chip structure, temperature and altitude variation on pressure sensors. A calibration check should be carried out at the chosen intervals for these pressure sensors to get accuracy. This paper helps to get information about how temperature and altitude affect the value of pressure and how sensor data is visualized to the user using android application. This analysis can be used for aerospace engineering application while airplane takes off or while landing.
\end{abstract}

Keywords- Piezoresistive pressure sensor, Micro electric mechanical system, Temperature, Altitude

\section{INTRODUCTION}

\section{A. Sensor Network}

The sensor is a device, module, or subsystem whose purpose is to locate occasions or changes in its surroundings and ship the data to other electronic devices such as computer with certain application software. The sensing material utilized in the piezoresistive pressure sensing element is diaphragm fashioned on the substrate that bends as pressure is applied. To spot sensitivity projected by sensing element, completely different structures of membrane are used like square and rectangular. The structure of membrane can offer a higher sensitivity compared to others need to be analyzed. Chip structure is a main part of sensing element. Sensors having different chip structure offer different sensitivity results.

\section{B. Pressure Sensor}

The pressure sensor is a tool to measure the pressure of gaseous, liquid atmospheric changes. Fig.1. shows pressure sensor.

Revised Manuscript Received on July 10, 2019.

Vaishnavi Wagh, Department of Information Technology, Walchand College of Engineering, Sangli, Mshsrashtra, India. (vaishnaviwagh26@gmail.com)

Shefali Sonavane, Department of Information Technology, Walchand College of Engineering, Sangli. Mshsrashtra, India.

(shefali.sonavane@walchandsangli.ac.in)

Chandan Kapoor, Baker Huges a GE Company, GE Oil \& Gas Ind Pvt Ltd, Mumbai. Mshsrashtra, India. (Chandan.Kapoor@bhge.com)

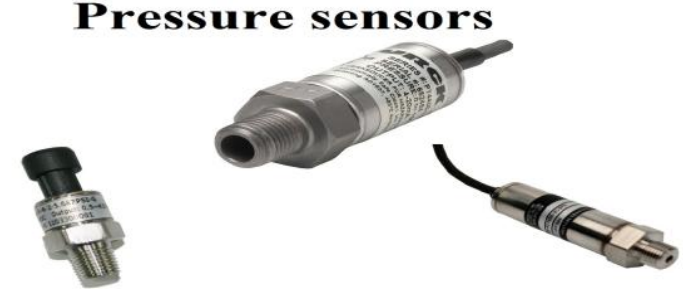

Fig.1. Pressure Sensors [13]

This pressure sensor can be used for any dimension of glide of fuel, liquid, speed, water level, altitude, and temperature. These sensors are particularly used in aerospace engineering in which pressure measurement plays an essential role. These pressure readings are important to guide the high height vehicle to take-off or landing. Another category of the pressure sensor is described to provide an explanation for excessive velocity adjustments in pressure. This pressure sensor particularly is built using piezoelectric cloth in particular quartz. One of the utility is altitude sensing which is utilized in aircraft, racket, satellite, climate balloons. This application tells the connection between changes in pressure with varying altitude and this relation is defined in equation 1.

$\mathrm{h}=\left(1-\left(\mathrm{P} / \mathrm{P}_{\mathrm{ref}}\right) 0.190284\right) * 145366$

This equation is graded for an altimeter as much as 36090 feet. Out of doors, range blunders can come out which is brought due to variation in temperature. The barometric pressure sensor is used with an altitude resolution of one meter that is enormous than GPS machine having 20 meters of altitude resolution. This altimeter can differentiate navigation among avenue stage and level of pressure readings. The pressure reading is calculated at different heights i.e. at different altitude to get results of pressure reading.

\section{TERPS (Trenched Etched Resonant Pressure Sensor)}

Trench Etched Resonant Pressure Sensors use a resonating silicon pressure-sensor which exploits the naturally occurring perfect elasticity of the single crystalline structure. TERPS is a resonant silicon pressure sensor innovation that gives a size which has more prominent precision and dependability than current pressure estimation advances. It is high precision sensor with $\pm 0.01 \%$ FS (Full Scale) over compensated temperature range. Fig.2. shows Trenched Etched Resonant Pressure Sensor. 
Features and benefits of TERPS are:

I. High Stability, $\pm 50 \mathrm{ppm}$ FS/year (typical).

II. Wide choice of pressure \& electrical associations to suit explicit necessity.

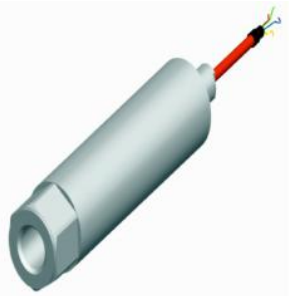

Fig.2. Trenched Etched Resonant Pressure Sensor [3].

Types of TERPS Sensor:

- TERPS 81XX

- TERPS 80XX

- TEPRS 82XX

- TERPS 83XX

\section{LITERATURE SURVEY}

A. Nallathambi, T. Shanmugantham, D. Sindhanaiselvi, "Design and Analysis of MEMS based Piezoresistive Pressure Sensor for Sensitivity Enhancement" [1] in 2018 described approximately strain deflection and sensitivity on square and rectangular membrane. The use of INTELLISUITE tool with a voltage of 8 volt provides high sensitivity and linearity of $0-1 \mathrm{MPa}$ for the same surface area. For rectangular and square shapes, sensitivity of $6.8 * 10-12$ for five $\mu \mathrm{m}$ thickness and $84.2 * 10-12$ for three $\mu \mathrm{m}$ thickness is determined. Rectangular membrane offer well matched running with a pressure range of $0.1 \mathrm{MPa}$ to $1 \mathrm{MPa}$. It uses finite element analysis modeling to simulate MEMS tool. End result had been located with the square diaphragm to get maximum pressure compared to the rectangular diaphragm. The limitation of this paper is that, the design and analysis of square and rectangular membrane are done for the low-pressure range from $0-1 \mathrm{MPa}$. This analysis is not suitable for pressure greater or smaller than $0-1 \mathrm{MPa}$. This paper does not provide any result for a circular membrane.

Meng Yuan, Ping Liu, Bo she, Youliang Tang, Yan Xu, "Research of MEMS Piezoresistive Pressure Sensor" [2] in 2010 described finite element method and one-of-a-kind simulation software which might be used with strain diaphragm of the piezoresistive micro pressure sensor. This paper is designed to get excessive voltage output with the help of two islands and beam combination which presents excessive sensitivity, precise linearity, and anti-overload. It defines resistor ought to be positioned on grooves of center and it's use. The limitation of this paper is that, this paper does not provide any results related to the resistivity of the sensor as this can be an important parameter to be considered for a piezoresistive pressure sensor.

Liwei Lin, Weijie Yun, "MEMS Pressure Sensor for Aerospace Application" [3] in 1998 defined fabrication technique that absolutely well matched with the integrated circuit. They used microelectronics with a single chip for each rectangular and circular-shape diaphragm having a thickness of two $\mu \mathrm{m}$ and width of one hundred $\mu \mathrm{m}$. This sensor is used with $100 \mathrm{psi}$ in complete scale span containing sensitivity of $0.15 \mathrm{mv} / \mathrm{v} / \mathrm{psi}$ and maximum linearity mistakes of $0.1 \%$ for complete scale span. Operational principle makes use of Wheatstone bridge type circuitry to offer voltage output for pressure measurement. It is observed that resistor have to be positioned at the radial position with a period of $2 \mu \mathrm{m}$ and oriented to the center. The limitation of this paper is that, parameters that are considered for design, modeling, and fabrication are not suitable to define the MEMS pressure sensor.

Xiaohui Du, Yifang Liv, Anlin Li, Zhou Zhou, Daoheng Sun, Lingyun Wang, "Laterally Driven Resonant Pressure Sensor with Etched Silicon Dual Diaphragm and Combined Beam" [4] in 2016 defined coupling mechanism of diaphragm and resonators. They used intercoupling between dual stress sensing diaphragm and laterally pushed resonant strain gauge. Pressure sensor is operating with a pressure variety of $30 \mathrm{KPa}$ to $190 \mathrm{KPa}$. However, it is far determined that sensitivity furnished with the aid of this coupling mechanism is poor and will increase as much as $8 \mathrm{~Hz} / \mathrm{KPa}$ and q-factor goes up to 20000 after water vacuum-packaging.

Myroslav Tykhan, Orest Lvakhiv, Vasyl Teslyuk, "New Type of Piezoresitive Pressure Sensors for Environments with Rapidly Changing Temperature" [5] in 2017 described effect of converting temperature on pressure reading. The pressure sensor with high accuracy and various temperature ranges are used in an aerospace application. They proposed that piezoresistive pressure sensor with the diaphragm can be utilized in high-velocity device. The limitation of this paper is that, all sensitivity results are found out by considering only one parameter e.g. coefficient of linear thermal expansion of the diaphragm. They did not give any result by considering other parameters of diaphragm like resistivity, thickness of diaphragm etc.

Budi Setiyono, Sumardi, Rafdito Harisuryo, "Measurement System of Temperature, Humidity and Air Pressure over $433 \mathrm{MHz}$ Radio Frequency" [6] described that with an increase in temperature there is a drop in pressure value and vice a versa. As this geographical constraint impact value of pressure, to keep away from this impact they call for some gadget which should be able to take care of all this statistics efficiently. It uses telemetry system for research outcomes. The distinctive form of sensors is used to get pressure information and to sense temperature and humidity for processing facts. The microcontroller is used to get statistics as it should be recorded. Acquisition gadget is evolved based on the user interface to discover adjustments in humidity and temperature. The limitation of this, paper is that this study does not use a real-time clock which is required to get correct reading value.

\section{PROPOSED WORK}

TERPS is a resonant silicon pressure sensor. The $8 \mathrm{XXX}$ series uses TERPS technology to measure atmospheric pressure. TERPS is having different properties and 
parameters to calculate pressure value. The 8000 Series uses trench etched resonant sensor technology. The RPS 8000 produces a frequency and diode voltage output. The DPS 8000 includes a microprocessor to produce a serial digital output. This research is carried out to visualize how sensor data is read and how it can be displayed through graphical user interface.

\section{A. Android Platform}

The android is a powerful operating system and it supports a large number of applications in smart-phones. These applications are more comfortable and advanced for use. Android application is created in order to visualize sensor data and sensor reading value.

\section{B. System Designing}

System design is the way toward characterizing the design, modules, interfaces and information for a framework to fulfill indicated necessities. Framework configuration could be viewed as the use of system design to accommodate advancement. The motivation behind the framework design is to enhance the framework for giving valuable data and information which is fundamental for the execution of the system components.

\section{Data Log Export}

Data received from the sensor is logged continuously and this collected data is exported to CSV file and shared through different sharing applications. Sensor data includes sensor serial number, pressure range, firmware version, com-port setting and calibration date along with aviation parameter QNH, QFF, QFE.

- QFE (Qantas Frequent Elevation): Pressure at the aerodrome elevation e.g. under certain circumstance.

- QNH (Query Nautical Height): Reads aerodrome elevation (height above MSL).

- QFF (Query Filed Flyer): Depends on temperature.

\section{Leak Test}

Leak test is a process to detect and measure the pressure difference. Leak test uses two types of time, test time and wait time. User can calculate leak rate for a container by using sensor and leak test feature in the application.

Wait Time: Wait time is time which is defined by the user to wait before the start of the actual leak test.

Test Time: Test time is a time where the actual leak test is performed. Once test time is initiated, first reading and the last reading of pressure value is collected in order to perform a leak test.

Leak rate: Leak rate is the total number of pressure readings obtained per unit time.

Leak rate is calculated as:

Leak rate $=\frac{\text { First } \text { Press Reading }- \text { Last Press Reading }}{\text { Test Time }}$

Above formula defines leak rate i.e. difference between first pressure reading and last pressure reading divided by test time.

Test accuracy is calculated as

Test Acc $=\frac{\text { Number of Samples Correctly Identified }}{\text { Total Number of Samples }}$
Above formula defines testing accuracy to find whether obtained leak rate is correct or not.

\section{E. Methodology}

Steps used to collect and store data obtained from sensor are defined below.

Step 1: Airfield pressure is measured by airfield technician.

Step 2: Drivers are used to connect the sensor with different devices through USB.

Step 3: Sensor is connected to mobile device.

Step 4: Android application is used to visualize pressure reading along with sensor data.

Step 5: Pressure reading along with sensor specification will be stored in CSV file.

Step 6: Analyze the performance of different sensors to compare pressure readings.

System architecture is shown graphically in Fig 3 .

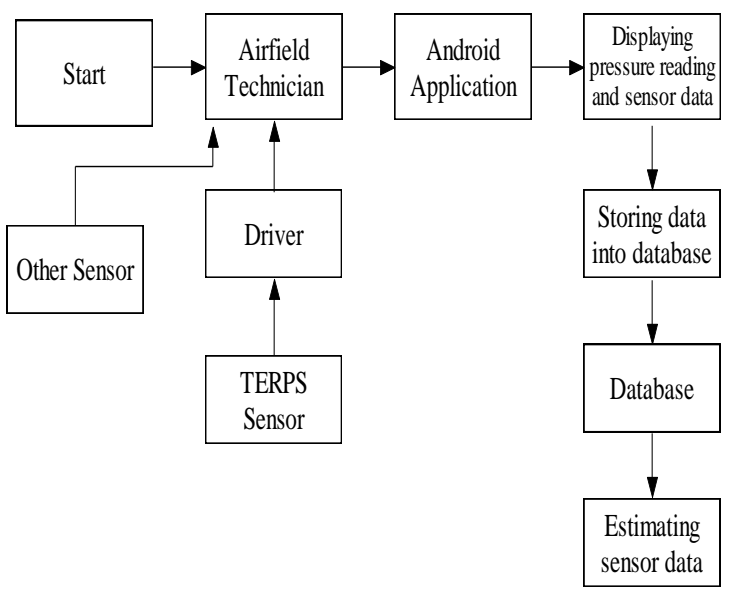

Fig.3. System Architecture

\section{F. Data Plotting}

Data received from the sensor is visualized through the plot. Per second data coming from the sensor is plotted. Data plotting contains two types of plotting, historical data plotting and live data plotting. Data plotting is used to show statistical data i.e. maximum, minimum, mean and standard deviation values which are calculated on collected sensor data. Sensor data is plotted with respect to time.

\section{RESULTS AND ANALYSIS}

Sensor data is collected by using android application and this sensor data along with aviation parameter is stored in a CSV file. This CSV file can be shared using different sharing application e.g. Gmail, Bluetooth, etc. 


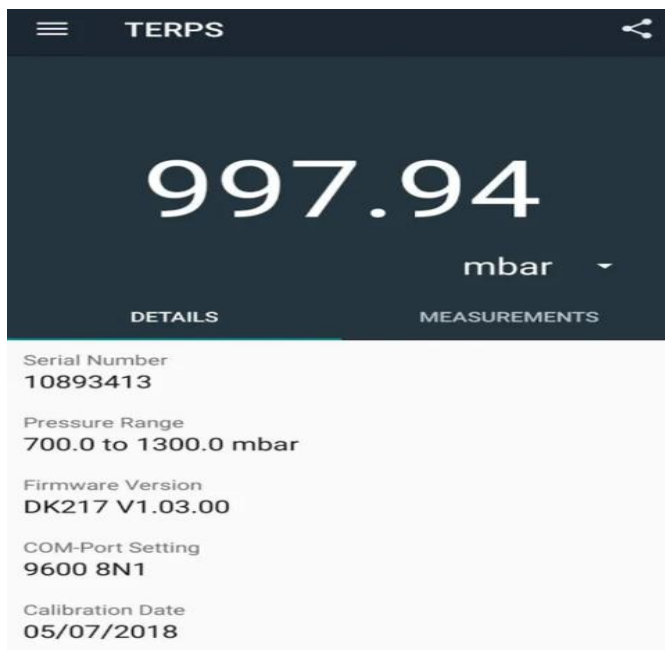

Fig.4. Sensor Parameters

Fig.4 shows the result of sensor data along with atmospheric pressure reading. Sensor data includes sensor serial number, a pressure range of the sensor, firmware version, and com-port setting. Each pressure sensor is having unique serial number and pressure range. Measurement tab contains information about all elevation parameter like QNH, QFF, and QFE along with values of altitude with respect to the atmospheric pressure. Fig. 5 shows generated CSV file of sensor data along with file name and file location. Sharing option is there to share the generated CSV file using different sharing application or through Gmail API.

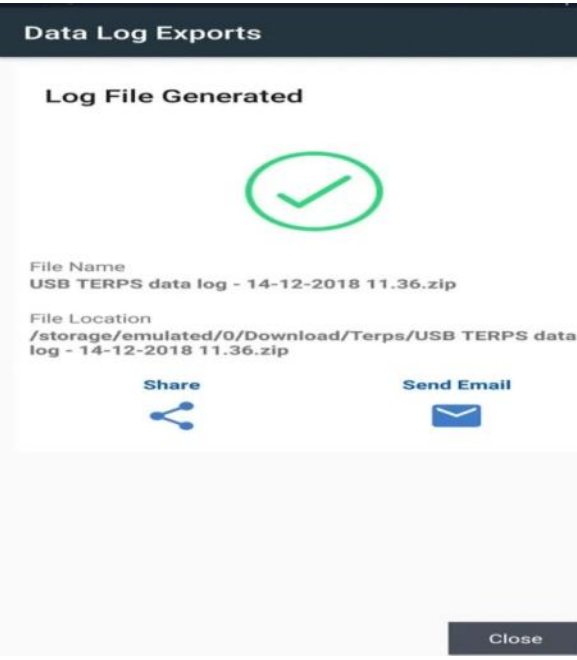

Fig.5. Sharing of sensor data
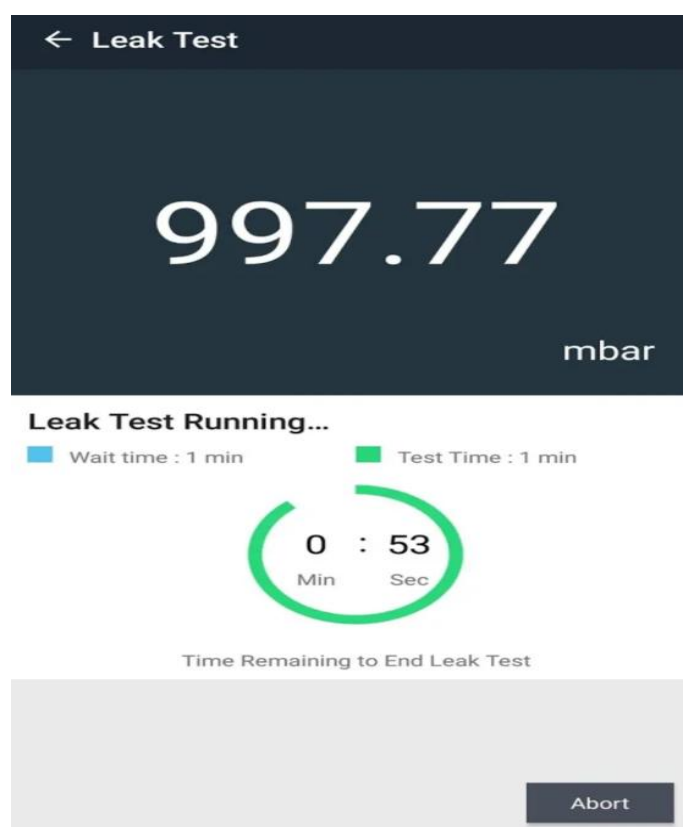

Fig.6. Leak Test implementation

Fig 6 shows leak test running and the time remaining for leak test. While operating with pressure sensors all time, it is necessary to find out leakage in order to get correct reading value from sensor.

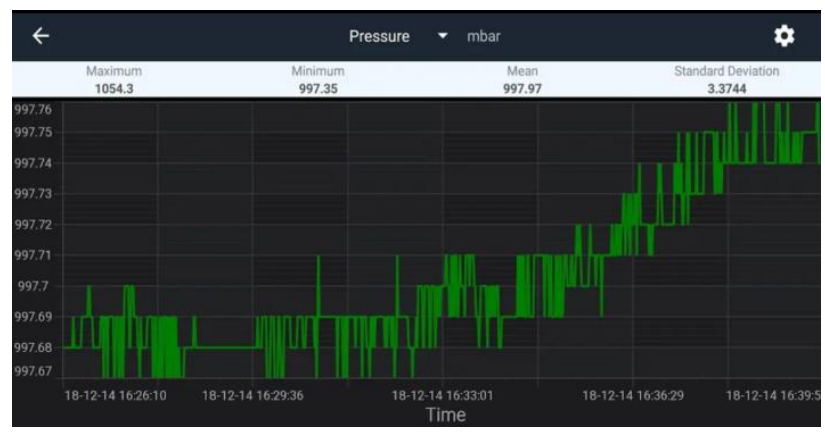

Fig.7. Historical data plotting

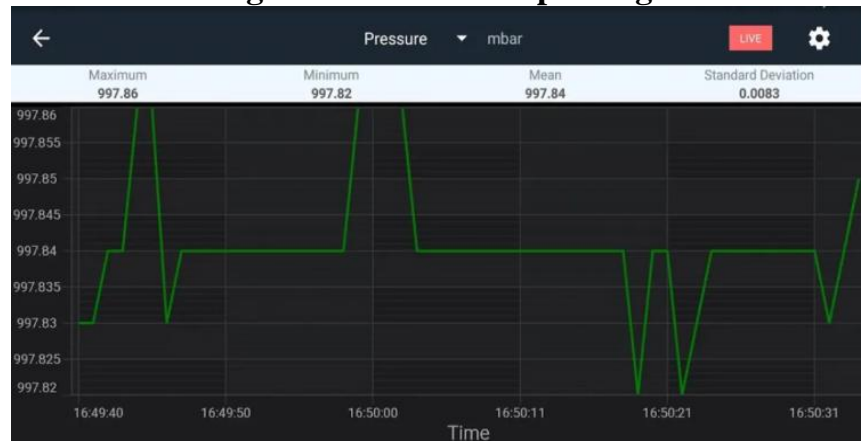

Fig.8. Live data plotting

Fig.7 and Fig.8 shows historical and live sensor data plotting. Historical data contains plotting of previously recorded sensor data. This plot is according to the date and time selected by user.

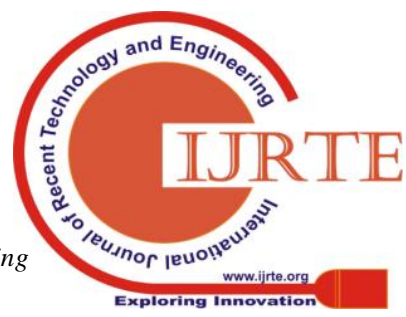




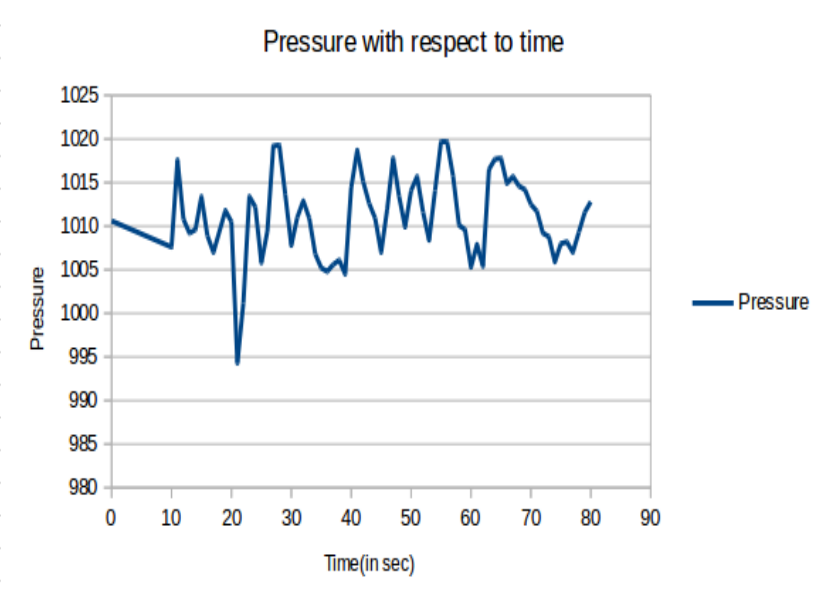

Fig.9. Pressure reading with respect to time

Fig. 9 shows the value of pressure with respect to time.

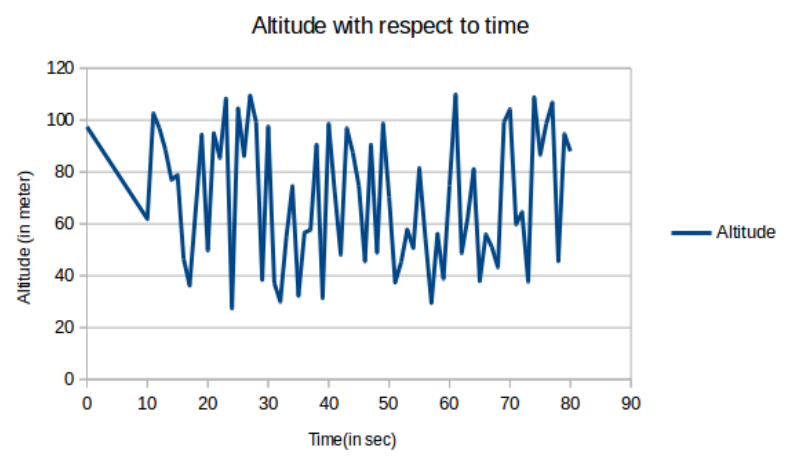

Fig.10. Altitude with respect to time

Fig. 10 shows the value of altitude with respect to time.

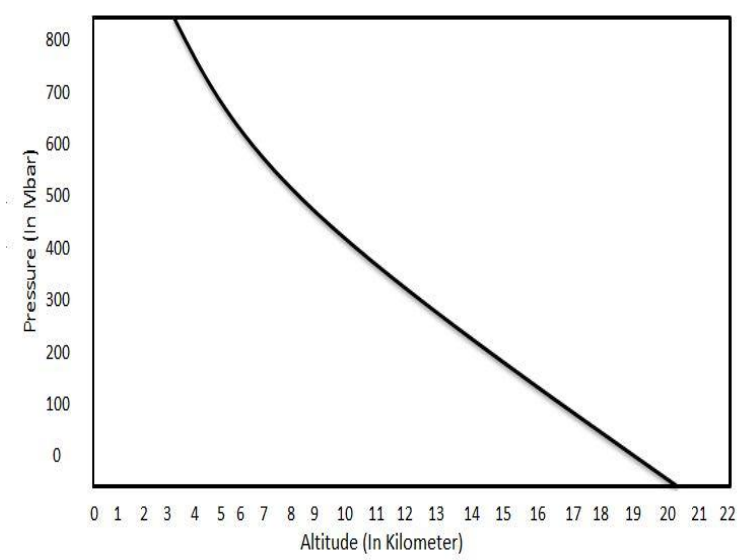

Fig.11. Relation between pressure \& altitude

Fig. 11 shows the inversely proportional relationship between pressure data and altitude data measured from mean sea level.

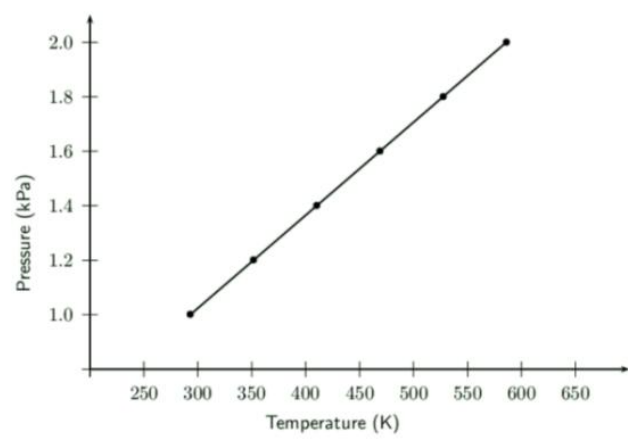

Fig.12. Relation between pressure and temperature.

Fig.12 shows the directly proportional relationship between pressure data and temperature.

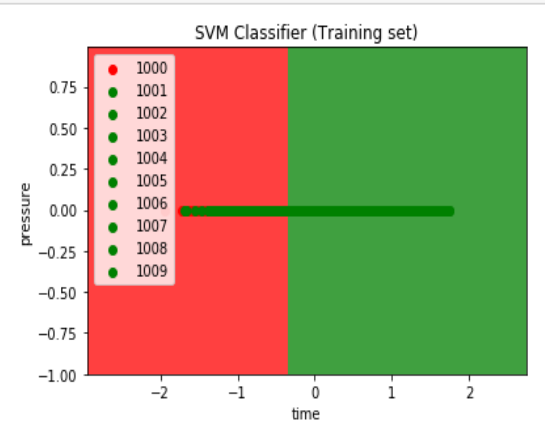

Fig.13. Classification of Sensor data using SVM.

Fig. 13 shows the classification result for sensor data set using support vector machine. This classification is used to predict the next pressure value.

\section{CONCLUSION}

This paper describes the pressure sensor application to visualize sensor data along with sensor specification using android system. The data received from sensor is stored and shared by android system. The impact of various parameters like temperature and altitude on pressure reading value are observed. Altitude is inversely proportional to pressure reading. This is because when pressure is calculated at higher elevation most of the air molecules remain at lower level towards earth due to gravitational force. Temperature is having direct proportional relation with atmospheric pressure. This is because higher temperature causes gas molecular to have greater kinetic energy. This causes high collision which leads to increase in pressure value.

\section{REFERENCES}

1. A. Nallathambi, T.Shanmugantham, D.Sindhanaiselvi, "Design and Analyse of MEMS based Piezoresistive Pressure Sensor for Sensitivity Enhancement" 2018 International Conference on Processing of Materials Minerals and Energy ,vol-5 ,pp 1897-1903 2018

2. Yuan, Meng Liu, PingShe, BoTang, Youliang $\mathrm{Xu}$, Yan "Research of MEMS piezoresistive pressure sensor" 2010 International Conference on Future Information Technology and Management Engineering, FITME,vol-536-539, 2010. 
3. R. Craddock \& P. Kinnell "Trench Etched Resonant Pressure Sensor” GE Measurement \& Control Solutions ,Fir Tree lane, Groby, UK LE6 0FH.

4. Lin, Liwei Yun, Weijie "MEMS Pressure Sensors for Aerospace Applications", 1998 IEEE Aerospace Conference Proceedings (Cat. No.98TH8339), 1998.

5. Du, XiaohuiLiu, YifangLi, AnlinZhou, ZhouSun, Daoheng Wang, Lingyun "Laterally driven resonant pressure sensor with etched silicon dual diaphragms and combined beams", Sensors (Switzerland).vol- 16, 2016.

6. M. Tykhan, O. Lvakhiv, V. Teslyuk, "New Type of Piezoresistive Pressure Sensor for Environments with Rapidly Changing Temperature", Metro. Measurement System. Vol24, pp. 185-192, 2017.

7. Budi Setiyono, Sumardi, Rafdito Harisuryo, "Measurement System of Temperature ,Humidity and Air Pressure over 433 MHz Radio Frequency "

8. International Conference on Information Technology, Computer and Electrical Engineering(ICITACEE) Indonesia, Oct 2018.

9. Qiao, Zhongtao Gao, FengqiLi, Qichang Wang, Guanglong "Design of Data Acquisition and Transmission System Based on MEMS Sensors" 2013 IEEE International Conference on Information and Automation (Icia), vol-921-926, Aug-2013.

10. Li, YanbingYuan, Meng Xu, Jiyong "A pressure sensor study and research", 2013 IEEE International Conference on Information and Automation (Icia) vol-255-258, Aug-2011.

11. Meti, ShwethaBalavald, Kirankumar B Sheeparmatti, B G "OPEN ACCESS MEMS Piezoresistive Pressure Sensor: A Survey” Int. J. Engin. Res. App., vol- 23-31, 2016.

12. Lammel, G.Armbruster, S.Schelling, C.Benzel, H.Brasas, J.Illing, M.Gampp, R.Se V.Schäfer, F.Finkbeiner, S. "Next generation pressure sensors in surface micromachining technology" Digest of Technical Papers - International Conference on Solid State Sensors and Actuators and Microsystems, TRANSDUCERS '05.vol- 35-36 , 2005.

13. "Pressure Sensor ", [Online] https://en.wikipedia.org/wiki/Pressure_sensor [Accessed:06 Oct 2018] 